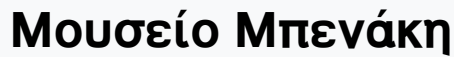

Tóp. 1 (2001)

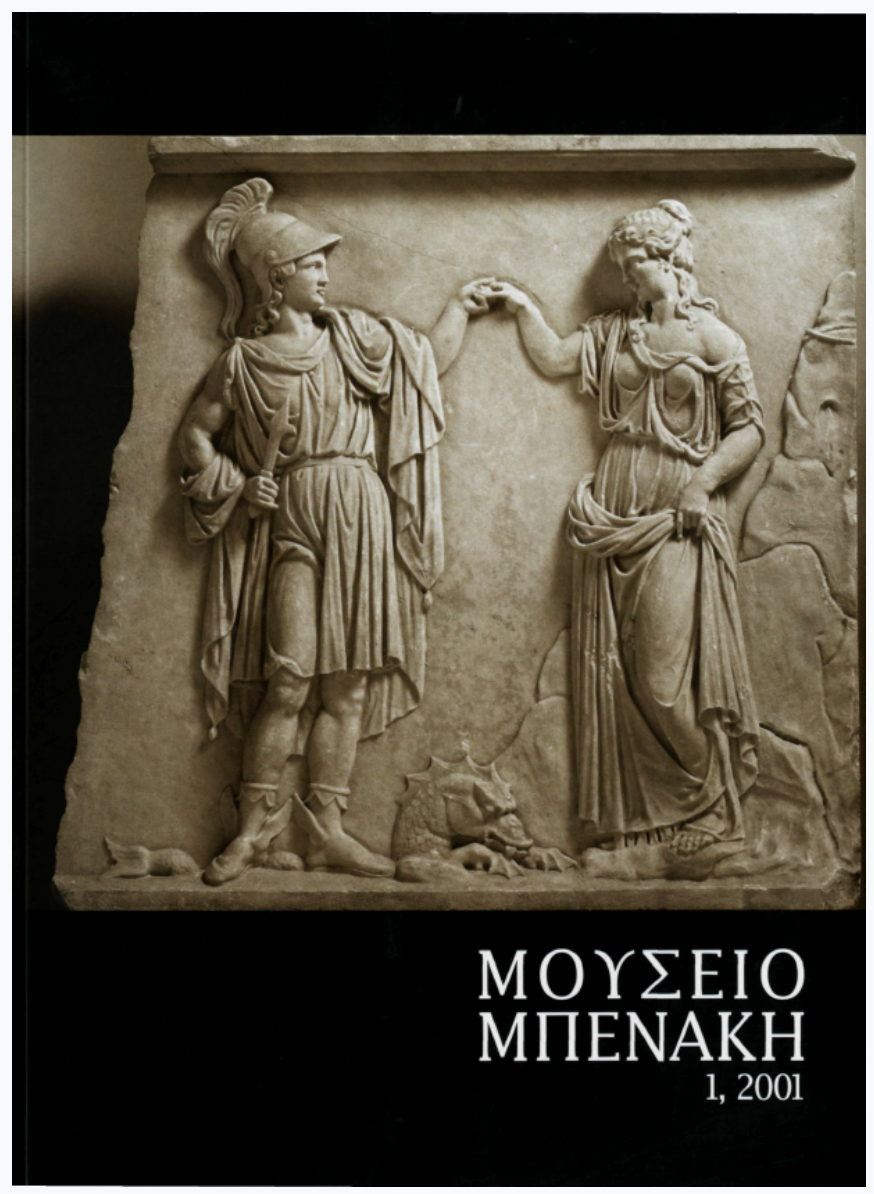

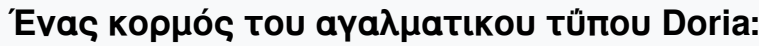

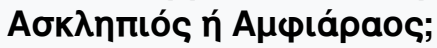

Marion Meyer

doi: $10.12681 /$ benaki.18321

Copyright @ 2018, Marion Meyer

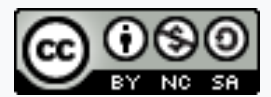

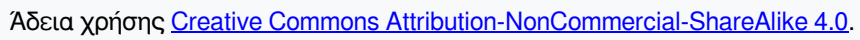

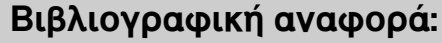

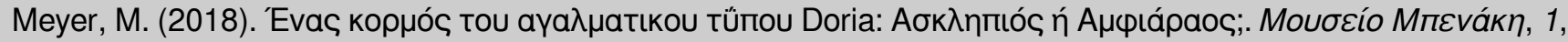
21-32. https://doi.org/10.12681/benaki.18321 


\section{Ein Torso im Typus Doria: Asklepios oder Amphiaraos?}

Als Geschenk der Familie Vallianos kam im Juni 1988 der männliche Torso einer Statuette aus pentelischem Marmor in das Museum Benaki (Abb. 1-4). ${ }^{1} \mathrm{Er}$ ist soweit erhalten, daß er als Darstellung einer Heilgottheit identifiziert werden kann. Die charakteristischen Merkmale sind das Stützmotiv und die Art der Manteldrapierung.

\section{Erhaltungszustand}

Abgebrochen sind der Kopf mit dem Hals, der rechte Fuß mit einem Teil des Unterschenkels, der linke Unterschenkel mit Fuß sowie der neben dem linken Oberschenkel herabhängende Teil des Mantels, wohl mit einem Teil des Stockes (der einst in die linke Achsel gestützt und weitgehend vom Mantel verdeckt war).

Es fehlen ferner der rechte Arm und das linke Handgelenk mit der Hand, die beide separat gearbeitet und angestückt waren. An den Ansatzstellen sind jeweils quadratische, $2 \mathrm{~cm}$ bzw. 2,4 cm tiefe Zapfenlöcher zu sehen. ${ }^{2}$

Neuzeitlich ergänzt waren der Kopf, der rechte Fuß mit dem fehlenden Teil des Unterschenkels und der neben dem linken Oberschenkel herabhängende Teil des Mantels. Die Brüche am Hals und am rechten Unterschenkel wurden für die vorgenommenen und wieder entfernten Ergänzungen geglättet. Die jeweils ovalen Löcher für die Anbringungen der Ergänzungen wurden nach ihrer Abnahme zugegipst.

Der Mantel weist Bestoßungen am Bausch und an der um den linken Arm gewickelten Partie auf, ferner geringfügige Abreibungen an verschiedenen Stellen. An der linken Brust befindet sich eine kleine Bestoßung.

\section{Beschreibung}

Das Gewicht des Körpers lastet auf dem rechten Bein und einem Stock, der schräg von der linken Achsel zur Seite geführt gewesen sein muß. Das linke Bein ist entspannt nach vorne genommen, der linke Fuß kann nur mit dem Ballen auf dem Boden aufgesetzt haben. Der linke, aufgestützte Arm ist etwas vom Körper abgespreizt und angewinkelt, so daß die Hand etwa in Höhe des Oberschenkelansatzes vor oder auf dem Stock zu liegen kam. Die Schulterlinie fällt stark zur Standbeinseite hin ab. Der rechte Arm war gesenkt und nach vorne genommen, wie aus der Beobachtung $\mathrm{zu}$ schließen ist, daß an der rechten Flanke der Figur keinerlei Spuren einer Stütze vorhanden sind. Wahrscheinlich hielt die vorgestreckte Hand eine Opferschale. Der Kopf war, den im Ansatz noch erkennbaren Halsmuskeln nach zu schließen, ein wenig nach links gedreht, zur Spielbeinseite hin.

Der schlanke, jugendlich wirkende Körper ist in einen Mantel gehüllt, der einen großen Teil des Oberkörpers unbedeckt läßt. Der obere Teil des Mantels bildet einen voluminösen Bausch, der sich vom linken Oberarm, die Schulter frei lassend, quer über den Rücken zieht, die rechte Hüfte gerade noch bedeckt, unterhalb des Nabels einen tiefen Bogen macht und steil zur linken Achsel hin ansteigt, wo er als Polster für den (nicht sichtbaren) Stützstock dient. An den Beinen liegt der Stoff verhältnismäßig eng an, so daß sich die Konturen beider Oberschenkel klar abzeichnen.

Die Oberfläche der Figur ist in gutem Zustand. Der einfühlsam wiedergegebene Körper mit seiner glatten, 


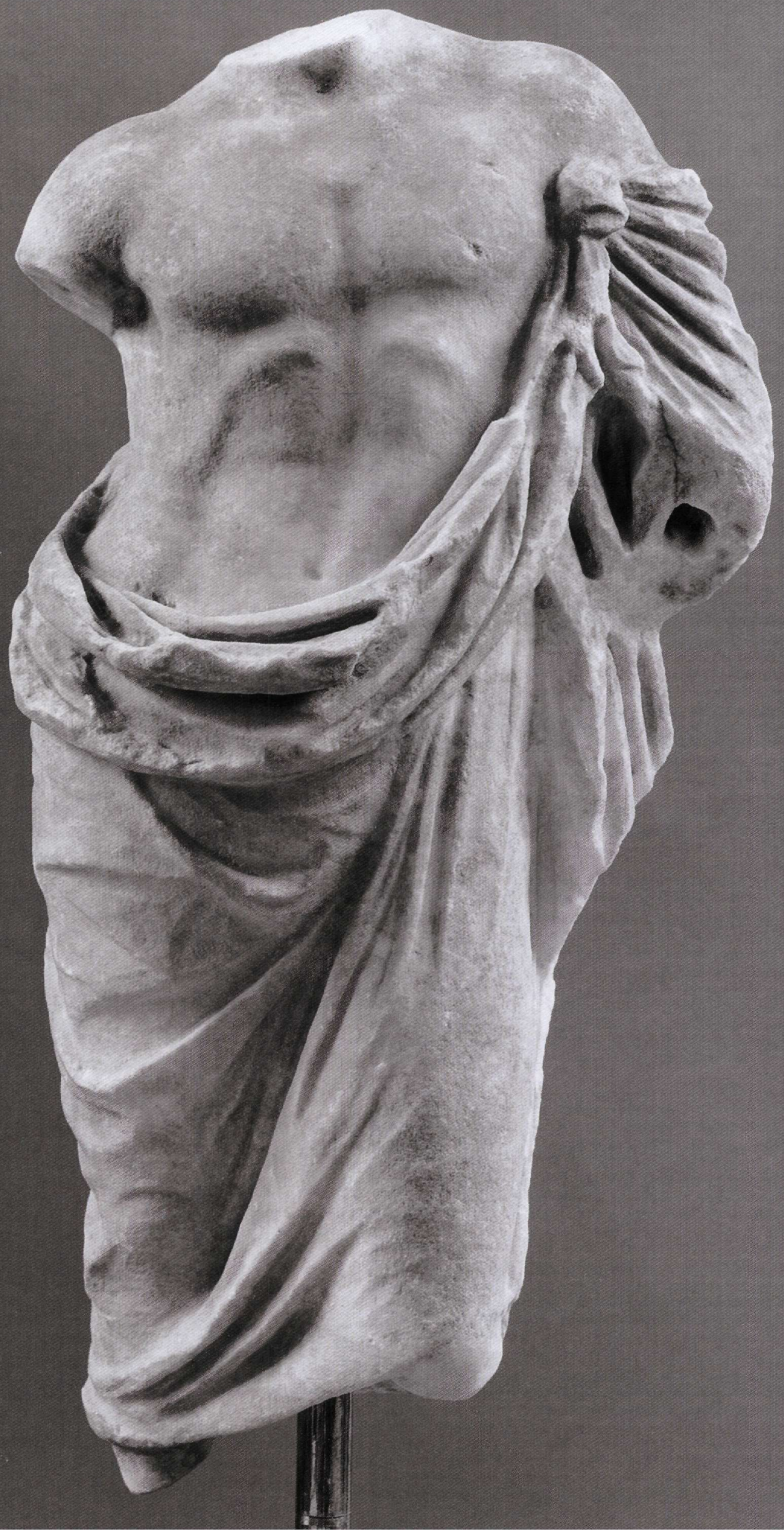



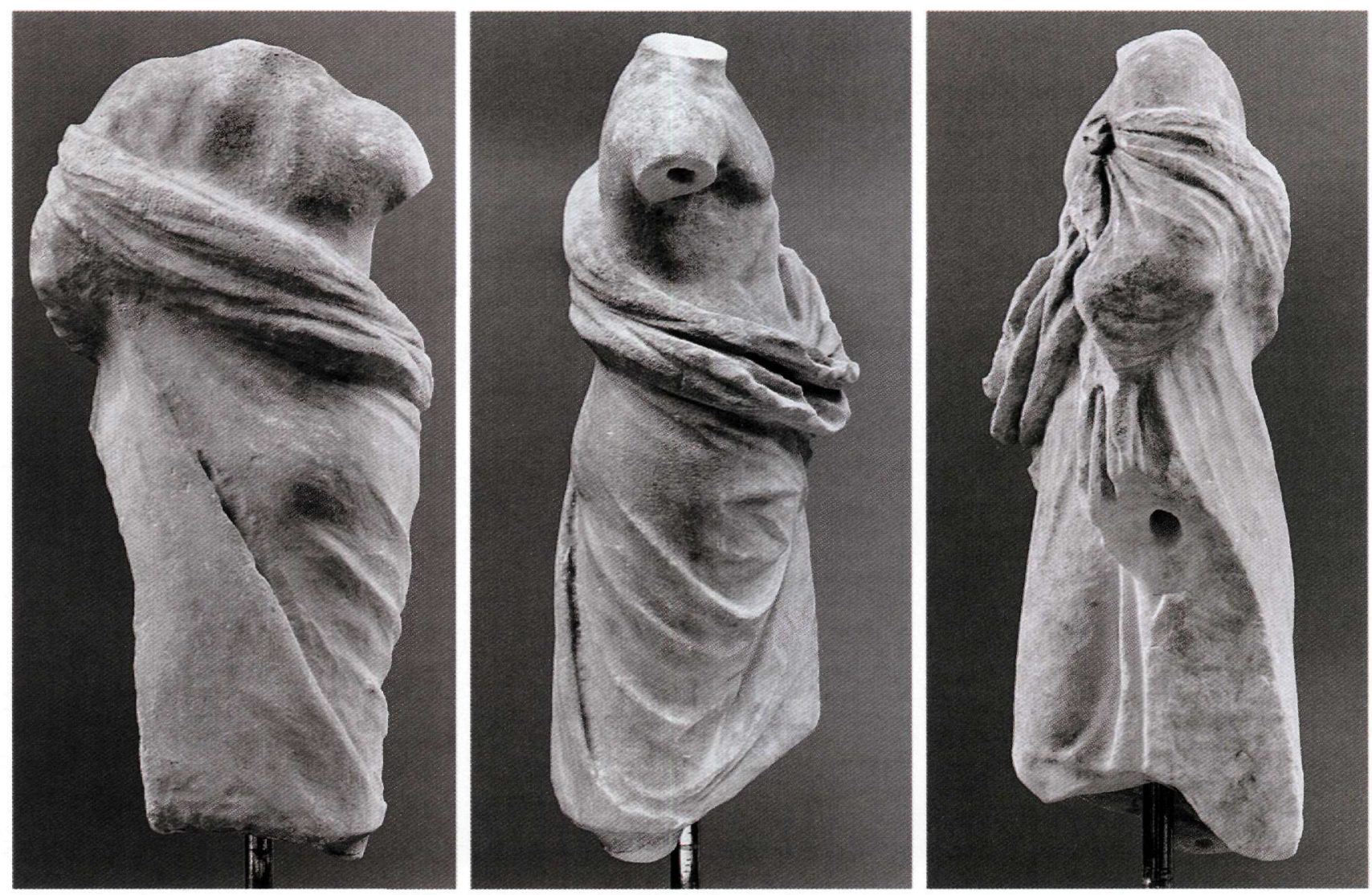

Abb. 1-4. Statuettentorso. Athen, Museum Benaki. 30245 (Foto: M. Skiadaresis).

weichen Haut bildet einen wirkungsvollen Kontrast zu dem kleinteilig bewegten Mantel mit seinen voluminösen Faltengraten und den tiefen, verschatteten Faltentälern.

Die Ausarbeitung der Statuette zielt auf die Wiedergabe von Bewegung und plastischen Werten ab. Den Körper durchzieht ein S-förmiger Schwung. Der Gott (oder Heros) neigt seinen Oberkörper stark zu seiner rechten Seite und biegt die Standbeinhüfte weit nach außen, so daß eine deutliche Einziehung in Taillenhöhe entsteht. Dadurch wird die Funktion des rechten Beines als Standbein betont. Das Spielbein hingegen ist deutlich entlastet und hängt locker durch; der Oberkörper ist auf dieser Seite gedehnt, die Schulter liegt höher als auf der Seite des Standbeines. Die Bewegung des Körpers wird durch die Gewandwiedergabe noch betont. Der Mantelbausch trägt an der rechten Hüfte stark auf und verbreitert die Figur beträchtlich (in der Rückansicht noch stärker als in der Vorderansicht). Das Stand- bein wird von flachen Bogenfalten umrundet, die die tragende Funktion dieses Beines verdeutlichen, während sich auf dem Spielbein der Stoff strafft.

Angesichts der geringen Größe der Statuette ist das Karnat bemerkenswert differenziert ausgearbeitet. Der Nabel ist als linsenförmige Einziehung in die leicht vorgewölbte Bauchdecke eingebettet. Die Rippenbögen zeichnen sich als breite Erhebungen weich unter der Haut ab. Die Medianlinie senkt sich verhältnismäßig tief ein, mit einer deutlichen Mulde oberhalb des Rippenbogens und einem abrupten Ende oberhalb der Brust. An der rechten Brust und der rechten Schulter staut sich weiches Karnat. Auf der Rückseite zeichnen sich die Schulterblätter in der ansonsten diffus bewegten Oberfläche markant ab.

Die Statuette war für eine Ansicht ausschließlich von vorne bestimmt. Ihre Rückseite wurde nur nachlässig ausgearbeitet, mit einem gestrafften Mantelbausch und grob angedeuteten Falten; das einzige ausgearbeitete 


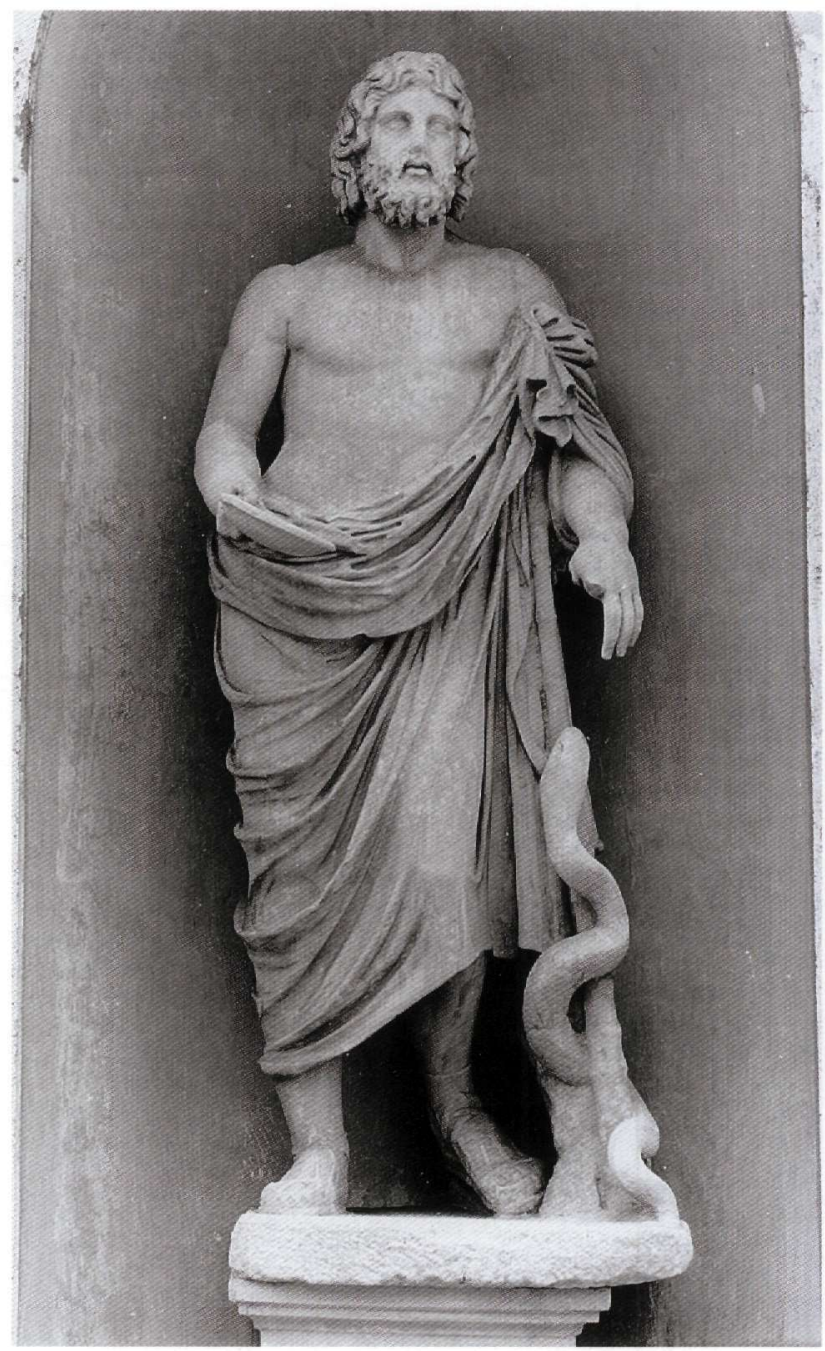

Abb. 5. Statue Rom, Villa Doria Pamphilj (Foto: DAI Rom InstNeg 69.496).

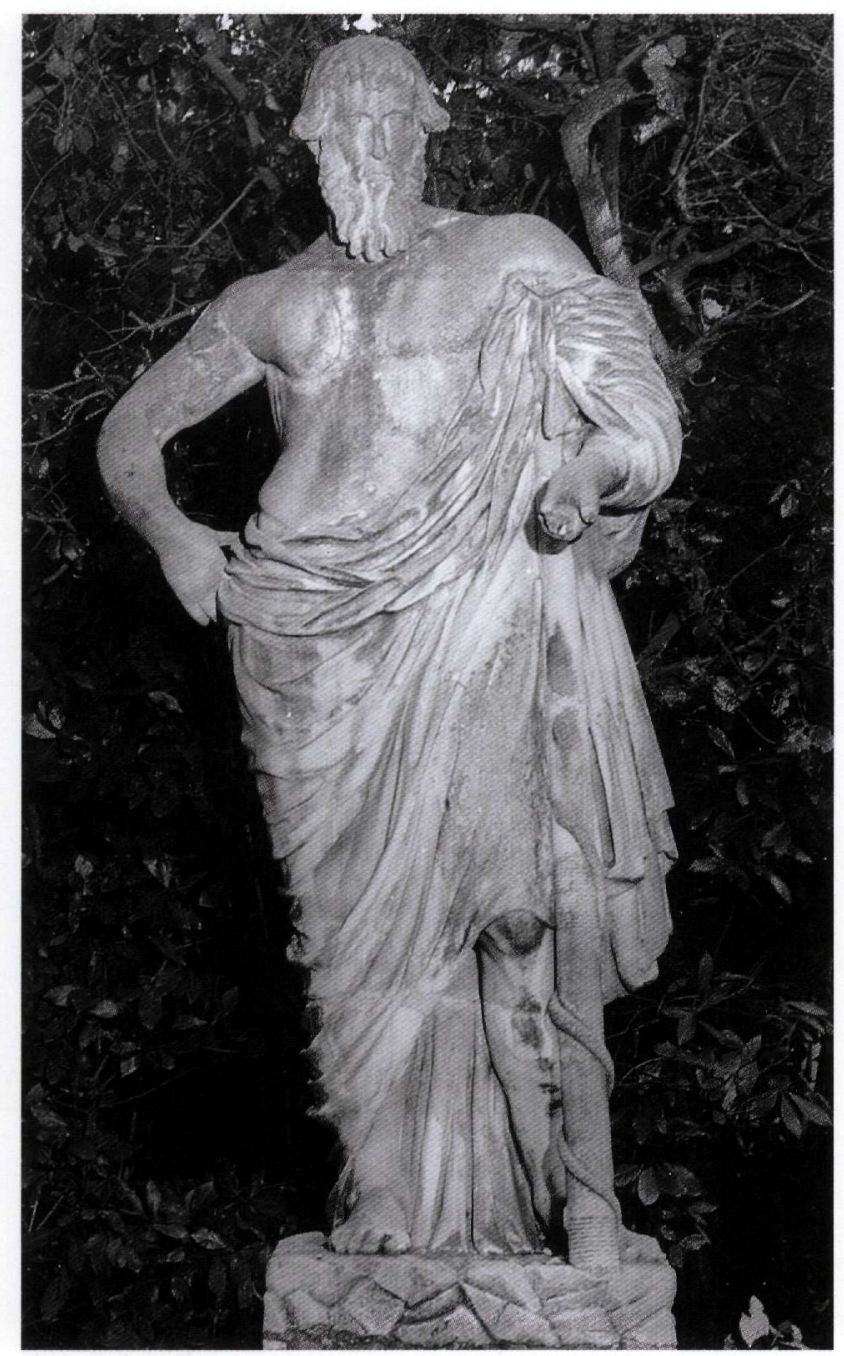

Abb. 6. Statue Florenz, Boboli-Gärten

(Foto: Gab. Fotografico, Soprintendenza beni artistici e storici di Firenze, No 329289).
Gewandmotiv ist die hinterschnittene Mantelpartie, die von der linken Taille quer über den Rücken zum Unterschenkel des Standbeins verläuft. Unter dem sich straffenden Gewand zeichnet sich die Rundung des rechten Glutäus ab.

\section{Entstehungszeit}

Die Wiedergabe des Körpers ist unverkennbar eine Reduktion und Beruhigung hochhellenistischer Formensprache. Die breiten, plastisch vortretenden Rippenbögen, die zur Mitte hin einschwingen, die dreieckige Mulde zwischen den Brüsten und den Rippen sowie die tiefe Medianlinie, die am Brustbein abrupt endet, findet man bei den nackten männlichen Oberkörpern der Figuren des Großen Altarfrieses von Pergamon. ${ }^{3}$ Dort treten diese Züge allerdings nicht so beherrschend in Erscheinung, weil die Brust-, Bauch- und Leistenpartien durchweg schwellend wiedergegeben werden und die Sägemuskeln stark hervortreten. Noch bei der Platte der sog. "Kleinen Gigantomachie", die stilistisch dem Telephosfries nahesteht, wölbt sich das Karnat stärker vor und ist kleinteiliger gegliedert als bei dem Torso Benaki. ${ }^{4}$ Dieser ist in der Verhärtung der Einziehungen bei gleichzeitig einfühlsam wiedergegebenem weichen Karnat (an der rechten Brust, Achsel und Schulter) dem gefallenen Gallier von Delos vergleich- 
bar, der um 100 v. Chr. angesetzt wird.

Die rechte Seitenansicht zeigt besonders gut, wie stark der massige Mantelbausch vor den Körper vorstößt und eigenes Volumen bildet. In der Vorderansicht besticht die Gewandwiedergabe des Bausches wie auch der eng die Beine umschließenden Mantelpartie vor allem durch die abwechslungsreiche Gestaltung von Faltentälern unterschiedlicher Form und abknickenden Faltengraten unterschiedlicher Höhe. Standbein und Spielbein werden durch ein langes, gerades Faltental getrennt; über dem Spielbein strafft sich der Stoff und legt sich eng dem Körper an, am Standbein staut er sich und bildet diffuse Bogenfalten. In der rechten Seitenansicht wird das Standbein durch zwei von der linken Körperseite kommenden, tief unterschnittenen Bogenfalten gerahmt, die die Massigkeit dieser Körperpartie betonen.

Hinsichtlich des Verhältnisses von Körper und Gewand wie auch der Wiedergabe des Mantels ist trotz des beträchtlichen Größenunterschiedes und anderer Proportionierung - die überlebensgroße Statue des Poseidon von Melos im Athener Nationalmuseum zu vergleichen, die im späten 2 . Jh. v. Chr. entstanden ist. ${ }^{6}$ Auch bei ihr besteht ein prononcierter Gegensatz zwischen dem voluminösen, die Hüftpartie verbreiternden und im Rücken hochgezogenen Mantelbausch und der eng um die Beine geschlungenen Gewandpartie, die sich auf dem Spielbein so stark strafft, daß kaum Falten entstehen, während das Standbein von Bogenfalten umrundet wird. ${ }^{7}$ Der Mantelstoff wird auch bei dem Poseidon von teils rinnenartigen, tief verschatteten, teils diffus in die Oberfläche eingetieften Faltentälern durchzogen. Eine entsprechende Gewandwiedergabe weist eine Asklepiosstatuette in Rhodos auf, die im Format eher dem Torso Benaki entspricht und ihm auch hinsichtlich des Verhältnisses von Körper und Gewand nahekommt. $^{8}$

Die Wiedergabe des Körpers wie des Gewandes erweisen den Torso als eine Arbeit späthellenistischer Zeit. Die Vergleiche sprechen für eine Datierung in die 2. Hälfte des 2. Jhs. v. Chr.

\section{Typologie}

Die statuarischen Darstellungen des Heilgottes Asklepios sind, gemessen an denen anderer Gottheiten, typologisch bemerkenswert einheitlich. ${ }^{9}$ Der Gott wird

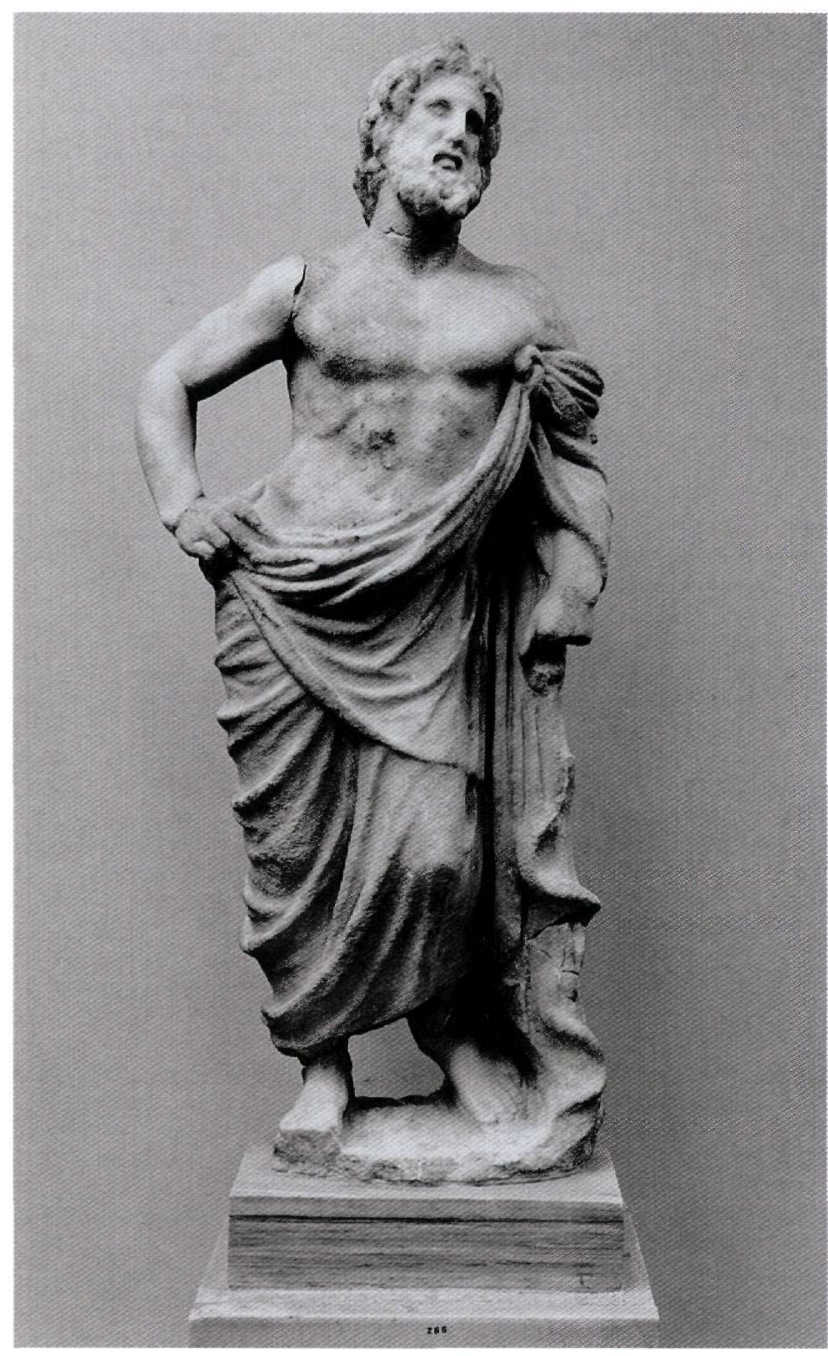

Abb. 7. Statuette Athen NM 266

(Foto: Athen, National Museum).

in der Regel stehend wiedergegeben, auf seinen Stock gestützt und mit einem Himation und stabilen Sandalen bekleidet. Es gibt nur eine verhältnismäßig geringe Variationsbreite hinsichtlich Ponderation und Manteldrapierung.

Aus dieser einheitlichen Überlieferung kristallisieren sich einerseits statuarische Typen heraus, die durch Replikenreihen belegt sind und deren Vorbilder u.U. auf bestimmte Kultbilder zurückgeführt werden können. ${ }^{10}$ Andererseits gibt es Statuen und Statuetten, von denen keine Repliken bekannt sind, die aber einzelne Motive mit einem oder mehreren statuarischen Typen gemeinsam haben bzw. auch Einzelmotive unterschied- 


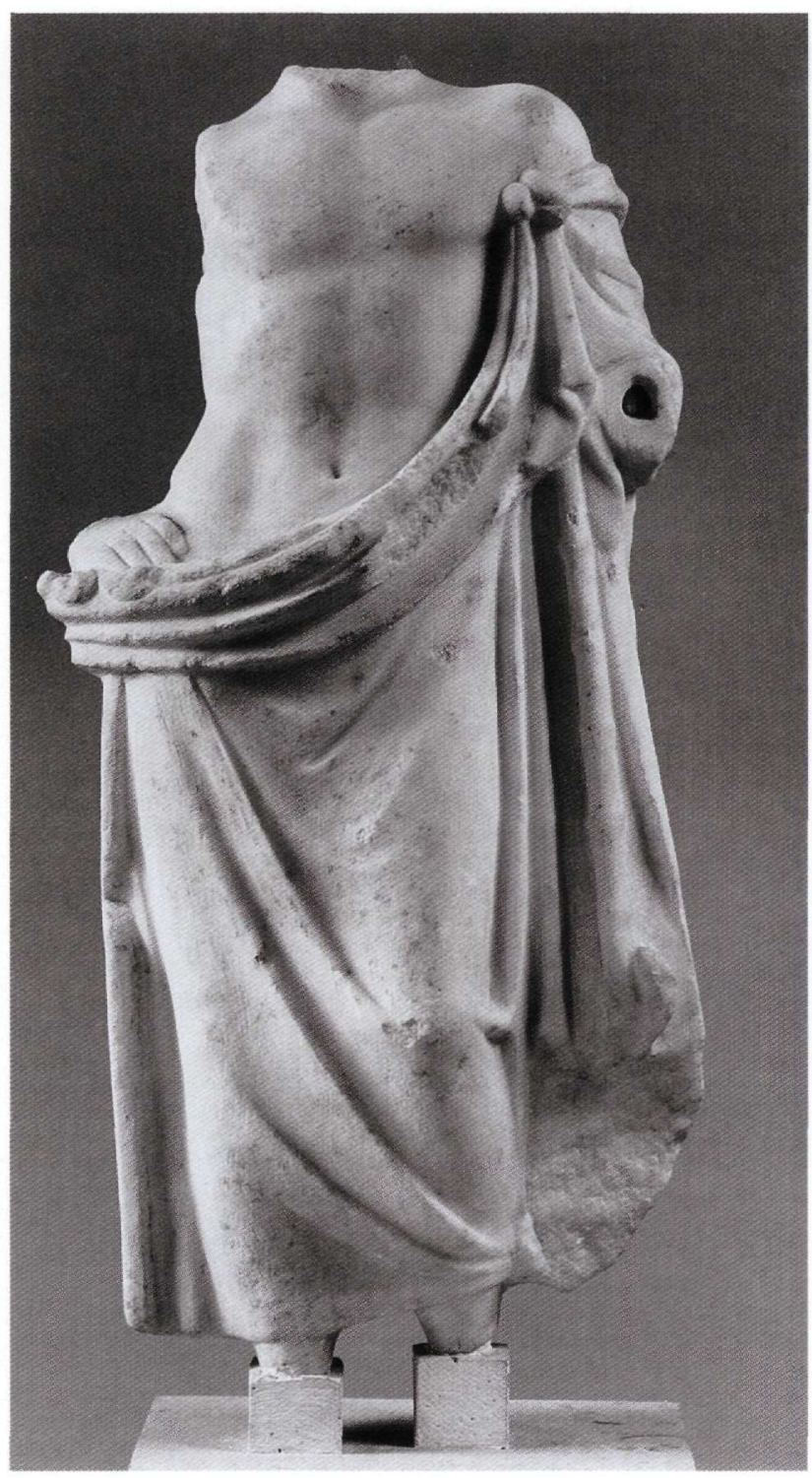

Abb. 8. Statuettentorso. Thessaloniki, Arch. Mus. 226

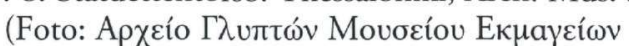

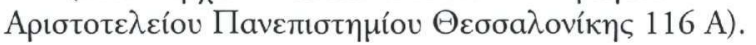

licher statuarischer Typen miteinander kombinieren können. Inwiefern es berechtigt bzw. sinnvoll ist, diese Werke als Varianten einem bestimmten Typus zuzuordnen, ist im einzelnen zu prüfen.

Der Torso Benaki gehört zu einem statuarischen Typus, der durch drei großformatige kaiserzeitliche Wiederholungen überliefert ist und als "Typus Doria" bezeichnet wird, nach dem Aufbewahrungsort des am besten erhaltenen Exemplars (Abb. 5; vgl. Abb. 6). ${ }^{11}$ Dieser Typus setzt sich von dem Gros der Heilgötter- darstellungen durch die knappe Manteldrapierung mit starker Entblößung des Oberkörpers ab. Der Mantel reicht auf der Standbeinseite bis zur Mitte des Unterschenkels und auf der Spielbeinseite bis zum Knie bzw. bis unterhalb des Knies. Er bedeckt gerade noch die Hüfte des Standbeins und läßt den Nabel frei. Auch die linke Schulter ist nackt; der Mantel ist bis auf den Oberarm herabgerutscht und wird nur durch den in die Achsel geklemmten Stock gehalten. Der obere Teil des Mantels bildet einen gleichmäßig breiten Bausch, der unter der linken Achsel eingeklemmt ist und dort zu Tütenfalten aufspringt. Der linke Oberarm hängt bis unterhalb des Ellenbogens in einer Mantelschlinge.

Von den drei Wiederholungen weichen die beiden des mittleren 2. Jhs. n. Chr. (in Rom und Florenz) in verschiedenen Zügen vom Typus ab. Bei dem frühkaiserzeitlichen Torso in Palermo wie auch bei der Statue in Rom (Abb. 5) war der rechte Oberarm gesenkt; der Unterarm muß jeweils nach vorne genommen worden sein, da sich keine Spuren der Hand oder einer Stütze an den rechten Flanken finden. Beiden Exemplaren ist ferner die das Spielbein eng umschließende Manteldrapierung bis unterhalb des linken Knies gemeinsam. In allen diesen Punkten weicht der Torso in Florenz (Abb. 6) ab. Nach antiken Ansatzspuren war bei ihm der rechte Arm abgewinkelt und die Hand in die Hüfte gestützt. ${ }^{12}$ Der schwere Mantel verhängt den Unterkörper vorhangartig, er zieht sich nicht eng um das Spielbein wie bei den beiden erst genannten Exemplaren. Dadurch entsteht ein gewisser Gegensatz zwischen dem breit wirkenden Unterkörper und dem schmalen Oberkörper. Der Mantel endet bereits in Kniehöhe. Bei der namengebenden Statue in der Villa Doria Pamphilj in Rom (Abb. 5) hingegen ist der Oberkörper weniger lang und schmal als bei den Wiederholungen in Palermo und Florenz (Abb. 6), und die Schulterlinie verläuft fast gerade.

Welche Kopfwendung der Typus vorgab, ist nicht mit Sicherheit zu entscheiden. Bei der Statue in Rom (Abb. 5) sitzt der Kopf ungebrochen auf; er ist erhoben und blickt leicht zu seiner linken Seite. Der Torso in Palermo hatte hingegen der Beobachtung von D. Arnold zufolge einen nach rechts, zur Standbeinseite hin gedrehten Kopf. ${ }^{13}$

Die für den Typus in Anspruch zu nehmenden Züge - der hochaufgewachsene, schlanke Körper, der S-för- 
mige Körperschwung und die auf Betonung der Ponderation abzielenden Gewandmotive mit ihrer klaren Unterscheidung zwischen eng das Spielbein umspielenden und breiten, horizontal das Standbein umfangenden Faltenbahnen - sprechen für eine Entstehungszeit im 4. Jh. v. Chr. ${ }^{14} \mathrm{Da}$ die Figur in ihren Konturen klar ablesbar ist und nur wenig räumliche Tiefe entwickelt, wird der Typus noch vor der Mitte des 4. Jhs. anzusetzen sein. ${ }^{15}$ Bei der mehrfach mit dem Typus Doria verglichenen Aphrodite Epidauros ${ }^{16}$ ist die Wiedergabe des Gewandes durch extreme Gegensätze zwischen Stoffpartien, die dünn und ohne eigenes Volumen auf dem Körper aufliegen und solchen, die sich vom Körper lösen (wie z.B. die Chitonpartie über dem rechten Oberarm) bestimmt, so wie bei den Skulpturen des Asklepiostempels in Epidauros aus dem frühen 4. Jh. v. Chr. ${ }^{17}$ Von diesen Werken setzt sich der Typus Doria durch eine insgesamt schlichtere, ausgeglichenere Formensprache $\mathrm{ab}$. $\mathrm{Zu}$ vergleichen sind Reliefs der Jahre um 370 v. Chr. mit Gewandfiguren, die gleichfalls eng in Mäntel mit diagonal den Körper überziehenden Faltenzügen eingewickelt sind..$^{18}$

Der Typus Epidauros, der verschiedene Motive mit dem Typus Doria gemeinsam hat (Standmotiv, Entblößung des Oberkörpers, Anlage der Gewandmotive im Beinbereich), ${ }^{19}$ ist hingegen stärker bewegt und entwickelt mehr Tiefe (s. Abb. 7). Der Oberkörper lehnt sich schwerer auf den Stützstock auf, so daß der Körper einen stärkeren Bogen beschreibt; das Spielbein greift weiter nach hinten aus. Der abgespreizte rechte Arm mit der an die Hüfte gelegten Hand trägt zur raumgreifenden Wirkung bei. Der Typus Epidauros wird im 3. Vtl. des 4. Jhs. v. Chr. entstanden sein. ${ }^{20}$

Der Torso einer Marmorstatuette in Thessaloniki, der in Olynth gefunden wurde und vor der Zerstörung der Stadt im Jahre 348 v. Chr. entstanden sein muß, hat mit dem Typus Doria das Standmotiv, die Entblößung des Oberkörpers und den gleichmäßig breiten Mantelbausch gemeinsam (Abb. 8). ${ }^{21}$ Die Motive im Bereich des Unterkörpers sind jedoch ganz unabhängig von diesem Typus gestaltet: der Mantel reicht weiter herab, die Zugfalten des Mantels verlaufen von der Standbeinhüfte zu Spielbeinknie und -unterschenkel, und das Standbein wird von Steilfalten gerahmt; ferner liegt die rechte Hand an der nackten Hüfte, oberhalb des Bausches. ${ }^{22} \mathrm{Da}$ die Statuette den Typus Doria

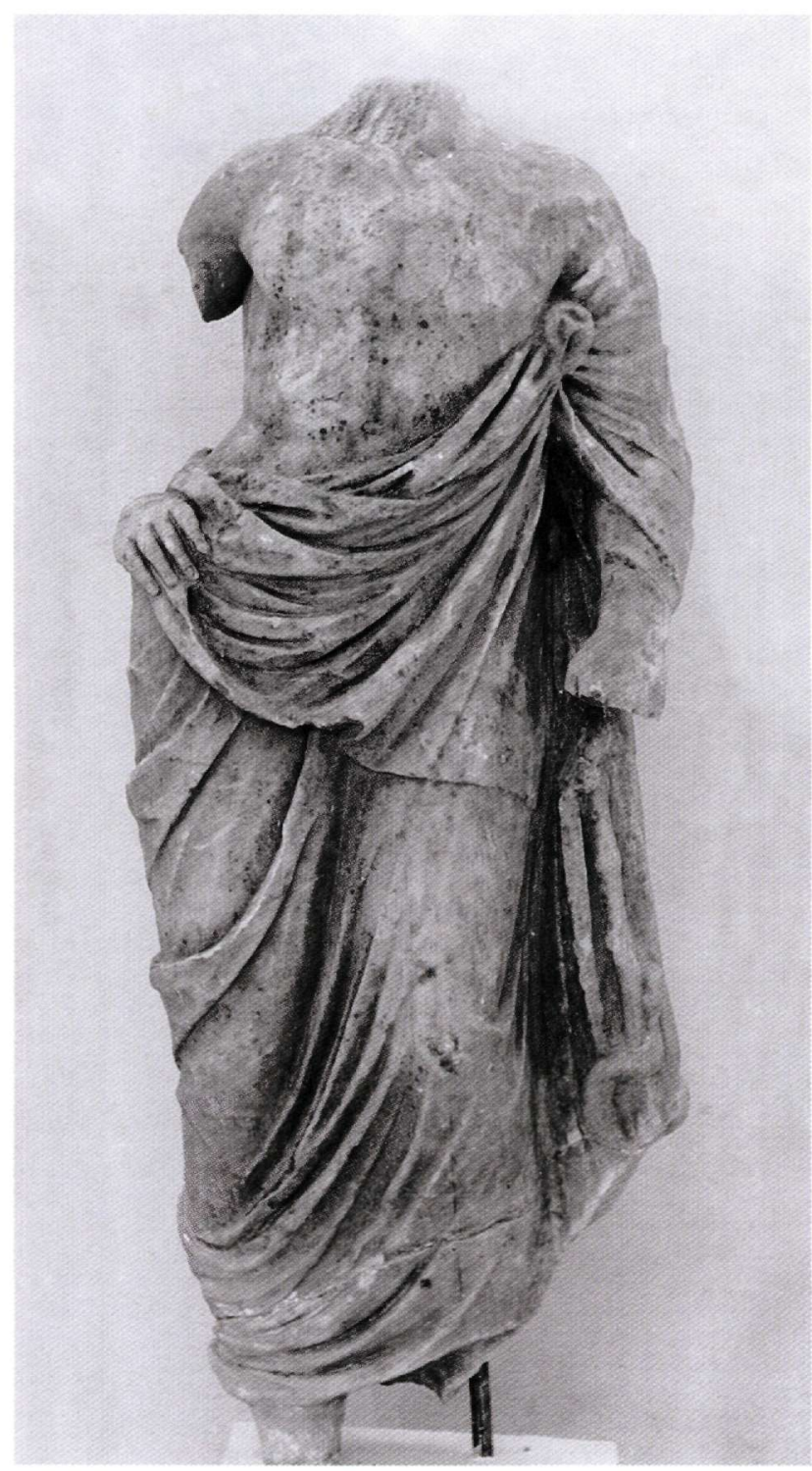

Abb. 9. Statuettentorso aus dem Amphiareion, Museum Oropos (Foto: Archäologische Gesellschaft zu Athen).

voraussetzt, ihm aber nur partiell folgt, ist sie als Variante zu bezeichnen. ${ }^{23}$

Der Torso Benaki ist das einzige hellenistische Werk, das dem Typus Doria zweifelsfrei zuzuordnen ist (während es diverse Wiederholungen des Typus Epidauros aus hellenistischer Zeit gibt). ${ }^{24}$ Die von diesem Typus abweichenden Züge erklären sich durch das Interesse des späten Hellenismus an Bewegung und plastischer Gestaltung. Die Schulterlinie fällt steiler ab, dadurch ist die rechte Körperseite insgesamt stärker 


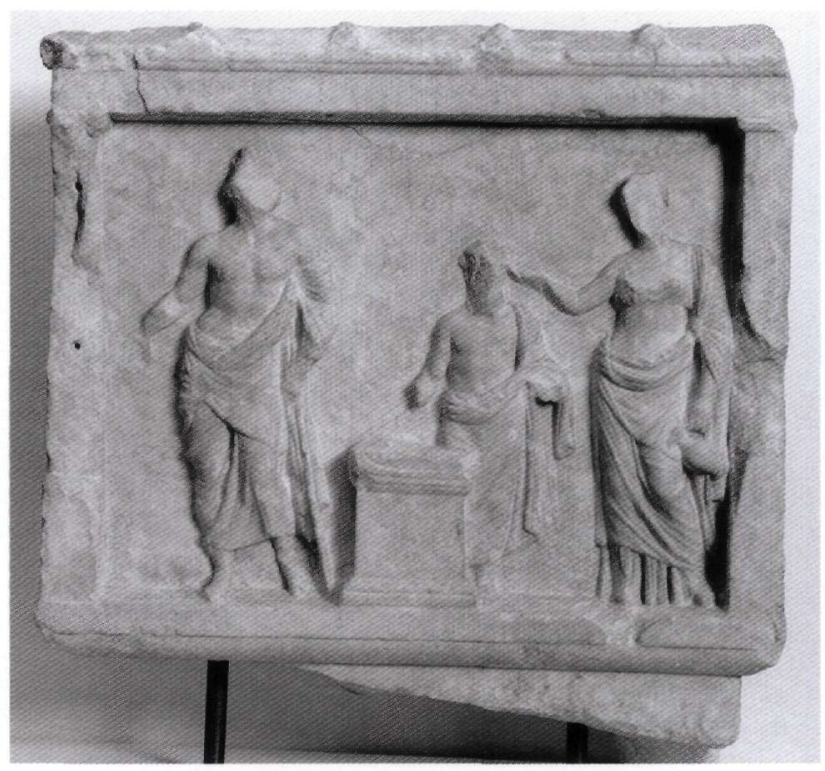

Abb. 10. Urkundenrelief Athen, NM 1396 (Foto: N. Stavritis).

gestaucht. Aus dem gleichmäßig breiten, flächigen Mantelbausch, der die Standbeinhüfte und die linke Achsel optisch akzentuiert, ist ein schwerer und voluminöser Mantelwulst geworden, der an der Hüfte deutlich breiter ist als an der linken Körperseite.

\section{Ergebnis}

Der Typus Doria war bisher nur durch die drei kaiserzeitlichen Wiederholungen in Italien überliefert (Abb. 5-6). Wenn die dem Typus zuzurechnende späthellenistische Statuette Benaki in Attika angefertigt wurde (wofür das Material, pentelischer Marmor, spricht), dann ist dies ein Indiz dafür, daß das im 2. Vtl. des 4. Jhs. v. Chr. entstandene Original ebenfalls in Attika stand. Ein zweites Indiz ist die Tatsache, daß die in Olynth gefundene Marmorstatuette, die den Typus Doria voraussetzt und kurz nach dem Original entstanden ist (Abb. 8), ebenfalls aus pentelischem Marmor gearbeitet wurde.

Der motivisch nahe stehende, jüngere Typus Epidauros (s.o. mit Abb. 7) wurde in Attika für die Darstellung des Heilheros Amphiaraos verwendet, wie der noch im 4. Jh. v. Chr. entstandene Statuettentorso aus dem Amphiareion in Oropos (Abb. 9) ${ }^{25}$ und ein Urkundenrelief von ca. $320 / 10$ v. Chr. (Abb. 10, mit einem variierten Figurentypus $)^{26}$ belegen. Das Original wird folglich ebenfalls in Attika zu lokalisieren sein. ${ }^{27}$ Aus dem Fundort des Urkundenreliefs (beim Hephaisteion) ist zu schließen, daß das Relief auf der Athener Agora aufgestellt war. Wenn der im Relief dargestellte und durch die Beischrift als Amphiaraos bezeichnete Heilheros den Figurentypus der Amphiaraosstatue aufnahm, die Pausanias auf der Agora sah, wäre das auf stilistischem Wege in das 3. Vtl. des 4. Jhs. datierte Original des Typus Epidauros ein guter Kandidat für ebendiese Statue. Ihre Aufstellung könnte durch die Rückgabe von Oropos an Athen durch Alexander motiviert und in den Jahren um 330 v. Chr. vorgenommen worden sein. ${ }^{28}$ Allerdings folgt die Figur des Reliefs dem Typus Epidauros nicht genau: die rechte Hand liegt nicht an der Hüfte, sondern ist mit einer Phiale vorgestreckt.

Die weitgehende Entblößung des Oberkörpers, wie sie der Typus Epidauros, aber auch der ältere Typus Doria vorgeben, erscheint für einen jugendlichen Heros ange-messener als für Asklepios selbst. So ist auf einem noch aus der 1 . Hälfte des 4. Jhs. v. Chr. stammenden Weihrelief der Heilheros Amphiaraos in einem anderen Typus, aber ebenfalls mit nackten Schultern zu sehen. $^{29}$

Bereits Heiderich warf die Frage auf, ob der Typus Doria, der als erster diese Entblößung vornimmt, ebenfalls den Heros Amphiaraos darstellt. ${ }^{30}$ Einziger Anhaltspunkt für diese Deutung ist eben diese Drapierung des Mantels, d.h. die motivische Gemeinsamkeit mit dem - später entstandenen - Typus Epidauros. Immerhin wäre zur Entstehungszeit des Typus Doria, d.h. um 370 v. Chr., durchaus mit der Möglichkeit zu rechnen, $\mathrm{da} ß$ in Attika eine Statue für diesen Heilheros aufgestellt wurde. In den Jahren zwischen 386 und 366 v. Chr. erlebte nämlich das an der Nordgrenze Attikas gelegene Amphiareion von Oropos, um dessen Besitz Athen und Theben stritten, unter attischer Herrschaft eine erste Blütezeit. ${ }^{31}$

Auch wenn sich nicht sicher klären läßt, welche der Heilgottheiten die Statuette Benaki darstellt - den Gott Asklepios selbst oder den Heilheros Amphiaraos -, so bereichert sie doch die Überlieferung beträchtlich: Sie ist der früheste Vertreter eines bisher nur aus kaiserzeitlichen Wiederholungen und einer Variante klassischer Zeit bekannten statuarischen Typus der Zeit um 370 v. 
Chr. Als attische Arbeit späthellenistischer Zeit legt sie nahe, daß ihr Vorbild, das Original des Typus Doria, in Attika aufgestellt war und sich auch in der 2. Hälfte des 2. Jhs. v. Chr. noch dort befand. Dieses Vorbild war anscheinend wenig berühmt oder bedeutend, denn es wurde weder auf einem der zahlreichen spätklassischen Weihreliefs für Heilgottheiten wiedergegeben noch

\section{A B K ÜR ZUNGEN}

Neben den im AJA 95, 1991, 4-16 empfohlenen Abkürzungen werden folgende verwendet:

Heiderich 1966: G. Heiderich, Asklepios (Diss. Freiburg 1966)

Holtzmann 1984: B. Holtzmann, in: LIMC II (1984) 863-97 s.v. Asklepios

Linfert 1990: A. Linfert, Die Schule des Polyklet, in: Poly- wurde es so häufig nachgebildet wie beispielsweise der motivisch ähnliche Typus Epidauros.

Prof. Dr. Marion Meyer

Archäologisches Institut der Universität

Am Hofgarten 21

D-53113 Bonn klet. Der Bildhauer der griechischen Klassik (Ausstellungskatalog, Liebieghaus, Frankfurt 1990, hrsg. von H. Beck u.a.) 240-97

Meyer 1989: M. Meyer, Die griechischen Urkundenreliefs (Berlin 1989)

Saladino 1991: V. Saladino, L' Asclepio del Giardino di Boboli, in: Boboli 90. Atti del convegno internazionale II (Florenz 1991) 595-607.

\section{A NMERKUNGEN}

1. Inv. 30245; Höhe: 0,405 m.; Fundort unbekannt.

2. Zur Anstückung des rechten Armes vgl. die entsprechende Zurichtung folgender späthellenistischer Werke: G. S. Merker, The Hellenistic Sculpture of Rhodes (Göteborg 1973) Kat. 69 Taf. 20 Abb. 48. 49; Kat. 70 Taf. 21. 22 Abb. 50-52. Zur Anstückung der linken Hand vgl. Merker (ibid.) 2 Kat. 52 Abb. 29-31 (= G. Gualandi, Sculture di Rodi, ASAtene 53, 1976 [1979] 90-91 Abb. 68).

3. AvP III, 2 (Berlin 1910) Taf. 3.8.9.11.12.14-16.19; W.D. Heilmeyer (Hrsg.), Der Pergamonaltar (Tübingen Berlin 1997) 80-85 mit Abb.

4. AvP VII,2 (Berlin 1908) 282-83 Nr. 356 Beil. 39; Heilmeyer (ibid. 36 Abb. 15).

5. J. Marcadé, Au musée de Délos (Paris 1969) 119-27 Taf. 80; A. Hermary u.a., Sculptures déliennes (Paris 1996) 20405 mit Abb. (mit Lit.).

6. J. Schäfer, Der Poseidon von Melos, AntP 8 (1968) 5567 Taf. 38-40; LIMC VII (1994) 452 Nr. 32 s.v. Poseidon (E. Simon). Zur Datierung Schäfer (ibid.) 59-60.64: 4. Vtl. des 2. Jhs. v. Chr.
7. s. bes. Schäfer (ibid.) 55-67 Abb. 9.

8. Rhodos E 520: Merker (Anm. 2) 12 Kat. 52 Abb. 2931; Gualandi (Anm. 1) 90-91 Abb. 68.

9. s. Holtzmann 1984, 868-89 (mit z.T. unbefriedigender Typenscheidung); A. H. Borbein, Zum ,Asklepios Blacas', in: Kanon. Festschrift Ernst Berger, 15. Beih. AntK (Basel 1988) 211.

10. Zum Asklepios Typus Giustini und davon abhängigen Typen: M. Meyer, Erfindung und Wirkung. Zum Asklepios Giu-stini, AM 103 (1988) 119-59; dies., Zwei AsklepiosTypen des 4. Jhs. v. Chr., AntP 23 (1994) 7-55; Zum Asklepios Typus Este und seinen Varianten: Borbein (ibid.) 211 mit Anm. 6; R. Kabus-Preißhofen, Die hellenistische Plastik der Insel Kos, 14. Beiheft AM (Berlin 1989) 31-51; Saladino 1991, 596-97 mit Anm. 17; zur bildprägenden Wirkung dieses Typus, vgl. P. Kranz, Die Asklepiosstatue im Schloßpark von Klein-Glienicke, JdI 104 (1989) 150. Zu diversen Asklepios-Typen: Kranz (ibid.) 107-55.

11. Replikenlisten: Heiderich 1966, 41-48.147-48 IV; D. Arnold, Die Polykletnachfolge (Berlin 1969) 177-78.274 M 1- 
3 (“Typus Girgenti”); Linfert 1990, 263-64.294 Anm. 75; Holtzmann 1984, 889 Nr. 373-78 (nicht als eigener Typus geschieden); Saladino 1991, 596-97: 1. Rom, Villa Doria Pamphilj (Abb. 5; ca. $2 \mathrm{~m}$ hoch; Kopf ungebrochen aufsitzend; rechter Unterarm mit Phiale ergänzt): EA 2333-2335; M. Bieber, A Bronze Statuette in Cincinnati and its Place in the History of the Asklepios Types, ProcPhilSoc 101 (1957) 80-81, Abb. 20-22; Heiderich 1966, 41-44.147 IV 1; Arnold (ibid.) 274 M 2 (hadrianisch-antoninisch); R. Calza (Hrsg.), Antichità di Villa Doria Pamphilj (Rom 1977) 55-56 Nr. 33 Taf. 24 (B. Palma); Holtzmann 1984, 889 Nr. 373; Linfert 1990, 294 Anm. 75 Nr. 2; L. Todisco, Scultura greca del IV secolo (Mailand 1993) 53 Abb. 45; Saladino 1991, 596 Abb. 188. - 2. Palermo 685 (aus Akragas): EA 553; Heiderich 1966, 42-43.148 IV 2; Arnold (ibid.) 274 M 1 (claudischflavisch); Linfert 1990, 294 Anm. 75 Nr. 1. - 3. Florenz, Boboli-Gärten (Abb. 6; 1,85 m.; ergänzt: Kopf, rechter Arm, linker Unterarm, Unterschenkel mit Füßen und Basis; große Teile des Stockes): EA 285; Heiderich 1966, 43.148 IV 3; Arnold (ibid.) 274 M 3 (antoninisch); Holtzmann 1984, 889 Nr. 374; Linfert 1990, 263-4.294 Anm. 75 Nr. 3, Abb. 129; Saladino 1991, 595-607 Abb. 187. - Nicht zugehörig: Linfert 1990, 294 Anm. 75 Nr. 6 (gehört zu Typus Epidauros); Holtzmann 1984, 889 Nr. 375 (s. Saladino 1991, 596 Anm. 8). - Zugehörigkeit fraglich, weil nicht in Abbildungen vorliegend: a) Heiderich 1966, 43.148 IV 4 (Wien, Slg. Lanckoronski); A. Schober, Asklepiosdarstellungen des vierten Jahrhunderts, Ö/h 23 (1926) 9 Anm. 10; Holtzmann 1984, 889 Nr. 363; b) Linfert 1990, 294 Anm. 75 Nr. 4 (Syrakus 6194); c) Linfert 1990, 294 Anm. 75 Nr. 7 (Oxford).

12. So P. Arndt, W. Amelung, Text zu EA 285 (München 1895); Heiderich 1966, 43. Anders Saladino 1991, 595-96 mit Anm. 7: rechte Hand ursprünglich nicht anliegend.

13. Arnold (Anm. 11) 178.

14. So schon P. Arndt, W. Amelung in den Texten zu EA 285.553 .

15. Vgl. etwa den Einschenkenden Satyrn des Praxiteles: Todisco (Anm. 11) 67 Abb. 101.102; C. Rolley, La sculpture grecque II (Paris 1999) 246-47 Abb. 240. - Für diese Datierung spricht auch die Statuette aus Olynth, die das Jahr 348 v.Chr. als terminus ante quem für den Typus vorgibt; s.u. mit Anm. 21-23.

16. Heiderich 1966, 46; Arnold (Anm. 11) 176-77 Taf. 20a; zustimmend Linfert 1990, 261-63 Abb. 123-125; zum Typus der Aphrodite Epidauros ferner: LIMC II (1984) 36 Nr. 243-44 s.v. Aphrodite (A. Delivorrias); J. Flemberg, Venus Armata (Stockholm 1991) 46-56 Abb. 1-4; Todisco (Anm. 11) 52 Abb. 43.

17. N. Yalouris, Die Skulpturen des Asklepiostempels in Epidauros, AntP21 (1992) passim; Rolley (Anm. 15) 203-07 Abb. 190-195. Diese Gegensätzlichkeit findet sich erstmals bei Skulpturen des Erechtheionfrieses: P. N. Boulter, The Frieze of the Erechtheion, AntP 10 (1970) 7-24 Taf. 1-30 bes. Taf. 1-3.10; M. Brouskari, The Monuments of the Acropolis (Athen 1997) 188-93 Abb. 130-133.
18. Urkundenrelief von 375 v.Chr.: Meyer 1989, 280 A 51 Taf. 16,2; C. L. Lawton, Attic Document Reliefs (Oxford 1995) 126-27 Nr. 96 Taf. 50; Münchner Lekythos: B. Vierneisel-Schlörb, Klassische Grabdenkmäler und Votivreliefs (München 1988) 121-27 Nr. 19 Taf. 42-44.

19. Namengebendes Exemplar: Athen, NM 266 (Abb. 6); Heiderich 1966, 72.149 VII 4a; Holtzmann 1984, 888 Nr. 361; Replikenlisten: Heiderich 1966, 41.70-71.149-50 VII 4; ferner: Sta-tuette aus dem Amphiareion von Oropos: Heiderich 1966, 47.148 IV 5 (= Holtzmann 1984, 888 Nr. 362); Holtzmann 1984, 888 Nr. 361-72 (davon nicht zum Typus gehörig: Holtzmann 1984, 888 Nr. 364.368.369; zu Holtzmann 1984, 888 Nr. 363 s.o. Anm. 11); Saladino 1991, 596 mit Anm. 15; Holtzmann 1984, Nr. 361 und Nr. 367 überliefern die Kopfwendung nach links, zum Stab und zur Spielbeinseite. Unterschiede zum Typus Doria: an die Hüfte gelegte rechte Hand; dreieckiger Mantelüberwurf, langer Mantel; diese drei Merkmale verbinden den Typus Epidauros wiederum mit dem Typus Este (zu diesem s.o. Anm. 10). Zum Typus Epidauros: Heiderich 1966, 70: Umbildung des Typus Doria; Holtzmann 1984, 888: Variante des Typus Este; KabusPreißhofen (Anm. 10) 45 mit Anm. 133: Variante des Typus, den sie für das Kultbild in Kos in Anspruch nimmt (d.h. des Typus Este, wobei Kabus-Preißhofen [ibid. 45-51] die namengebende Statuette für eine "Weiterbildung" hält).

20. Hinsichtlich der Körperhaltung und der räumlichen Wirkung ist der um 340/30 v.Chr. entstandene Statuentypus des 'Meleager' gut zu vergleichen: Todisco (Anm. 11) 87 Abb. 151; Rolley (Anm. 15) 274-75 Abb. 277; zum Typus: Ch. Vorster, Römische Skulpturen des späten Hellenismus und der Kaiserzeit I (Mainz 1993) 63-66 Nr. 25. Vgl. ferner den Körperschwung des Deloptes auf dem Urkundenrelief von 329/28 v.Chr. (Meyer 1989, 296 A 107, Taf. 32,2; Lawton [Anm. 18] 104-05 Nr. 47 Taf. 25; M. Moltesen, Greece in the Classical Period. Ny Carlsberg Glyptotek [Kopenhagen 1995] 138-41 Nr. 73 mit Abb.) und den der männlichen Hauptfigur auf einem etwas späteren Relief (Meyer 1989, 306 A 142, Taf. 42,1; Lawton [Anm. 18] 146 Nr. 150 Taf. 79).

21. Thessaloniki, Inv. 226: Bieber (Anm. 11) 82 Abb. 1718; Heiderich 1966, 151 VII 5 U 2; Linfert 1990, 294 Anm. 75 Nr. 5; Holtzmann 1984, 889 Nr. 377; Saladino 1991, 596 Anm. 13; G. Despinis u.a., Catalogue of Sculpture in the Archaeological Museum of Thessaloniki I (Thessaloniki 1997) 49-50 Nr. 29 Abb. 58-61 (G. Despinis).

22. Bei den - später entstandenen - Typen Este und Epidauros liegt die rechte Hand ebenfalls an der Hüfte, aber jeweils auf dem Gewand. So auch bei Heilgottheiten auf attischen Weihreliefs, seit der Einführung des Kultes in Attika um 420 v.Chr.; frühestes Exemplar s. E. B. Harrison, A Classical Maiden from the Athenian Agora in: Studies in Athenian Architecture, Sculpture and Topography Presented to Homer A. Thompson. Hesperia Suppl. 20 (1982) 44-45 Taf. 6d; C. Lawton, Votive Reliefs and Popular Religion in the Athenian Agora: the Case of Asclepios and Hygieia in: R. F. Docter, E. M. Moormann (Hrsg.), Proceedings of the XVth International Congress of Classical Archaeology Amsterdam 1998 (Amster- 
dam 1999) 233; ferner: Athen, NM 1332. 1334. 1341. 1345. 1375. 2487. 2926: J. N. Svoronos, Das Athener Nationalmuseum I (Athen 1908) 247-64 Nr. 29. 31. 38. 42 Taf. 36,2; 38. 34. 35; II (1911) 293 Nr. 72, Taf. 40,5; III (1937) 644.65556 Nr. 379.409, Taf. 154.186.

23. Vgl. Linfert 1990, 294 Anm. 75: "Originalvariante".

24. Zum Typus Epidauros s.o. mit Anm. 19. Zu diesem Typus und nicht zum Typus Doria gehören auch drei hellenistische Tor-sen, die mit den Typen Doria und Epidauros das Standmotiv und die Entblößung des Oberkörpers bis zur rechten Hüfte und linkem Oberarm gemeinsam haben: 1. Kalymnos (überlebensgroß): Linfert 1990, 294 Anm. 75 Nr. 8; Kabus-Preißhofen (Anm.10) 44.307 Nr. 103 mit Anm. 132: um 230 v.Chr. - 2. Rhodos. Museum E 173 (Statuette): Holtzmann 1984, 889 Nr. 378 mit Abb.; Kabus-Preißhofen (ibid.) 45 Anm. 135: späthellenistisch. - 3. Pergamon (Statuette): Holtzmann 1984, 889 Nr. 376. - Nr. 1 und Nr. 2 weisen das für den Typus Epidauros charakteristische Stützmotiv auf: die rechte Hand liegt mit gespreizten Fingern an der vom Bausch bedeckten Hüfte auf. Bei Nr. 1 ist außerdem der Ansatz des Mantelüberschlags unter dem Bausch zu erkennen (bei Nr. 2 fehlt dieser Überschlag). Auch bei Nr. 3 war der rechte Arm abgespreizt, also wohl aufgestützt; wegen des lang herabreichenden Mantels mit dem Überfall ist der Torso mit dem Typus Epidauros zu verbinden.

25. Erhaltene Höhe: 0,98 m; Holtzmann 1984, $888 \mathrm{Nr}$.

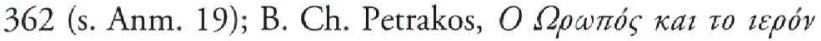
rov Apurajáov (Athen 1968) 120 Taf. 34b; LIMC I (1981) 701.710 Nr. 54 mit Abb. s.v. Amphiaraos (I. Krauskopf); Saladino 1991, 596-97 mit Anm. 16

26. NM 1396: Meyer 1989, 24.104-05.202-03.231.306 A 143, Taf. 42,2; Lawton (Anm. 18) 147-48 Nr. 153 Taf. 81; LIMC (ibid.) 702.710 Nr. 65 mit Abb.; Saladino 1991, 596-97 mit Anm. 16.

27. Nur von zwei weiteren der insgesamt sieben Wiederholungen des Typus Epidauros ist der Fundort bekannt: Epidauros (für das namengebende Stück, s. Anm. 19) sowie das Poseidon-Heiligtum auf Kalaureia (Heiderich 1966, 150 VII 4f; Holtzmann 1984, 888 Nr. 365).
28. Oropos hatte seit 366 v. Chr. zu Theben gehört; 335 v.Chr. wurde es von Alexander an Athen zurückgegeben; 322 v. Chr. verlor Athen es wieder; RE XVIII,1 (1939) 1173-74 s.v. Oropos (J. Wiesner); Petrakos (Anm. 25) 25-30; J. D. Mikalson, Religion in Hellenistic Athens (Berkeley 1998) 30 mit Anm. 46; 33 mit Anm. 15; 102.249.291. Zur Amphiaraosstatue auf der Athener Agora: Paus. I 8,2; LIMC (Anm. 25) 701 Nr. 57. Ihre Datierung in die Zeit vor $366 \mathrm{v}$. Chr. (so: U. Hausmann, Kunst und Heiltum [Potsdam 1948] 24) beruhte auf der fälschlichen Annahme von F. Kutsch, das Urkundenrelief stamme aus dem frühen 4. Jh. v. Chr. und belege einen Amphiaraoskult in Athen selbst bereits in dieser Zeit, s. F. Kutsch, Attische Heilgötter und Heilheroen (Giessen 1913) 39-47. Aus der lykurgischen Periode sind mehrere athenische Inschriften erhalten, die die Bedeutung des Amphiaraoskultes in dieser Zeit belegen, s. Mikalson (Anm. 28) 26.33-34.45.291.

29. Petrakos (Anm. 25) 124 Taf. 44; LIMC (Anm. 25) 702.710 Nr. 64 mit Abb. Allerdings kommt eine dem Typus Doria entsprechende Entblößung des Oberkörpers auch bei einem Gott auf einem Weihrelief aus dem Athener Asklepieion vor: NM 1373; Svoronos II (Anm. 22) 291-92 Nr. 70 Taf. 46.

30. Heiderich 1966, 46-47 hatte in dem Original des Typus Doria das Kultbild des Amphiaraos in Oropos sehen wollen, allerdings mit unzureichenden Gründen: die von ihm angeführte Statuette aus Oropos (s. Anm. 25) gehört dem Typus Epidauros an; das Urkundenrelief (s. Anm. 26) stammt nicht aus dem Amphiareion, sondern aus der Stadt Athen.

31. RE (Anm. 28) 1173-74; Petrakos (Anm. 25) 23-25; J. Travlos, Bildlexikon zur Topographie des antiken Attika (Tübingen 1988) 301-02 s.v. Oropos. Zur Bautätigkeit in Oropos zuletzt: H. R. Goette, Beobachtungen im Theater des Amphiareion von Oropos, AM 110 (1995) 253-60.

\section{MARION MEYER}

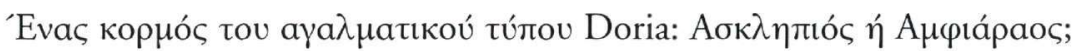

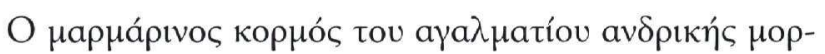

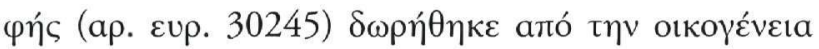

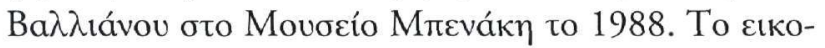

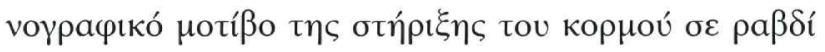

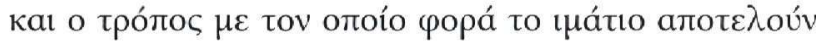

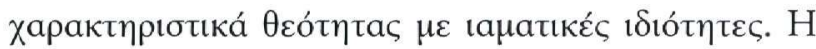


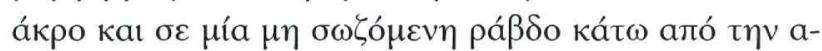




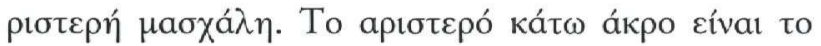

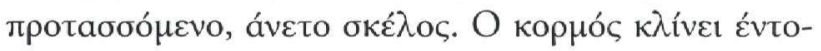

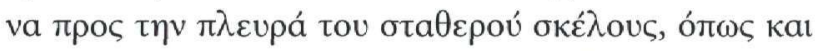

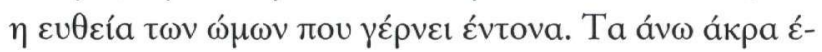

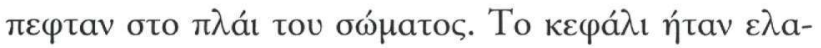

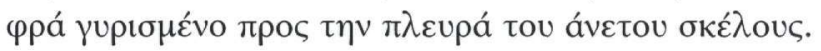

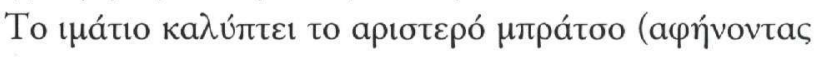

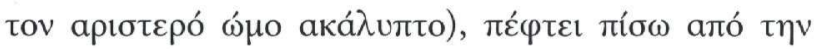

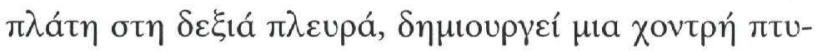

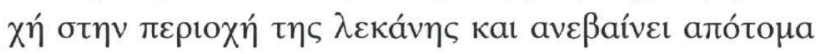

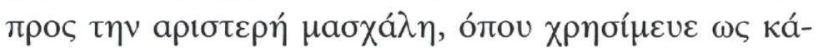

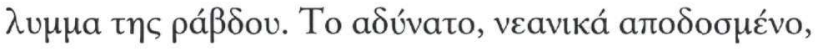

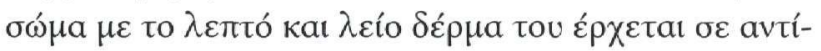

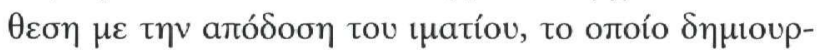

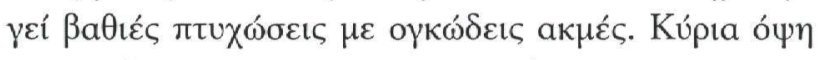

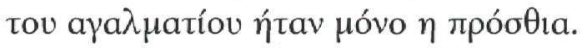

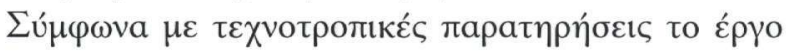

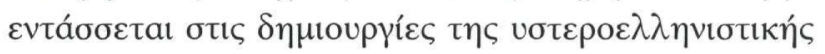

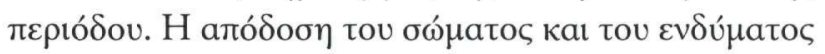

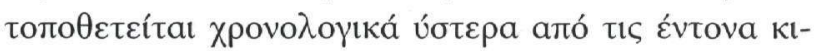

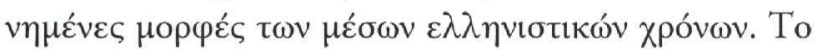

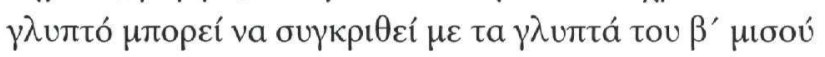
tov 2ov at. $\pi . X$.

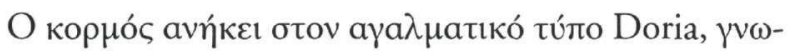

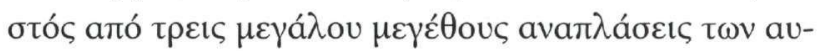

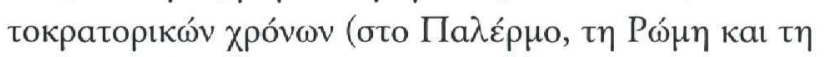

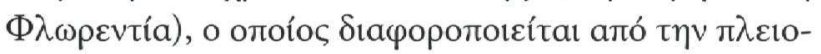

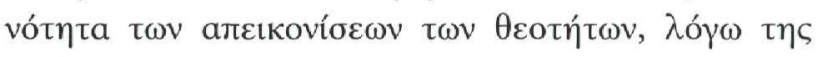

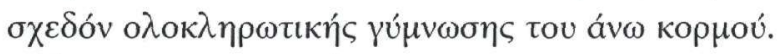

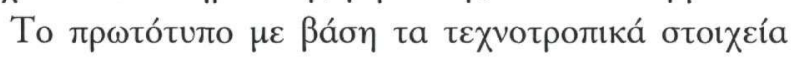

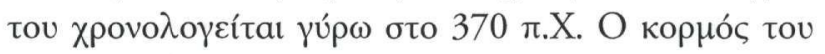

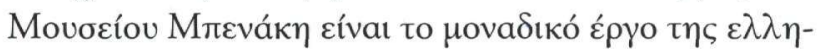

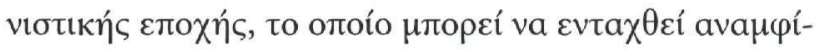

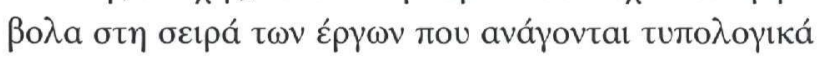

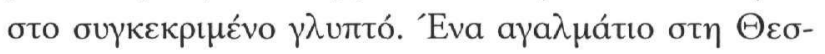

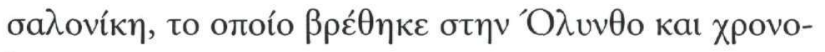

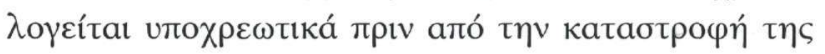

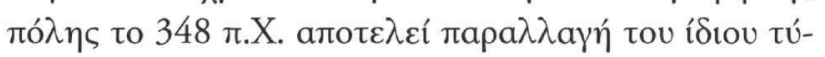

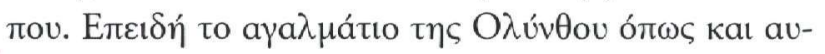

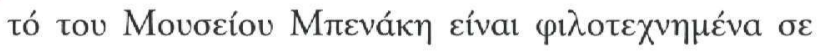

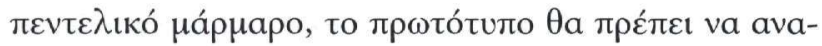

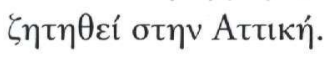

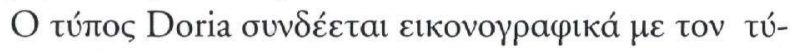

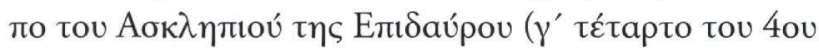

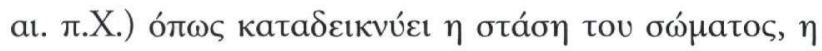

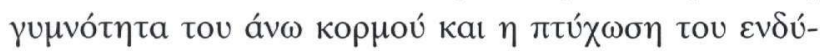

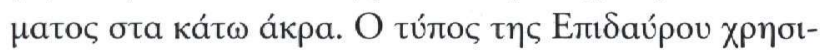

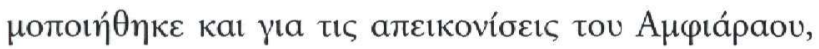

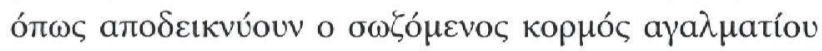

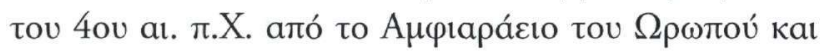

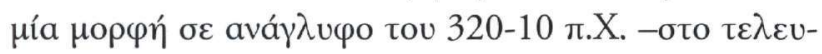

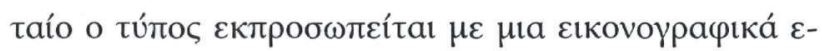

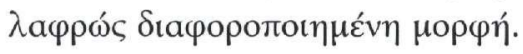

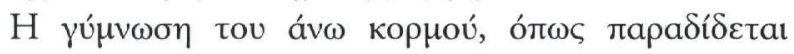

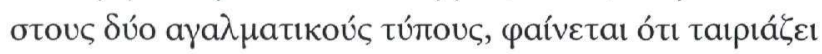

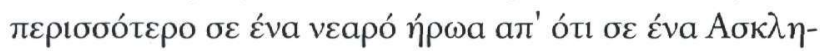

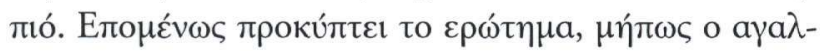

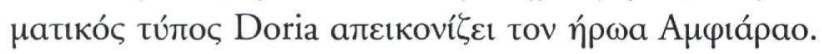

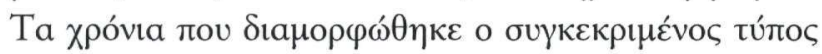

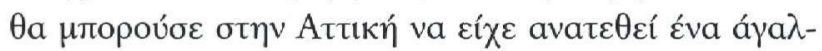

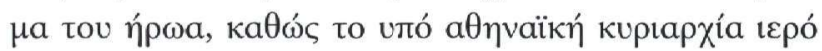

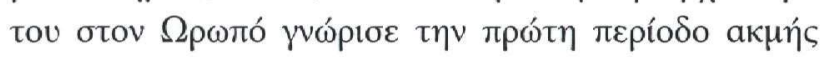
$\mu \varepsilon \tau a \xi \tilde{u}$ tou 386 кa $366 \pi$.X.

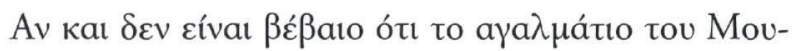

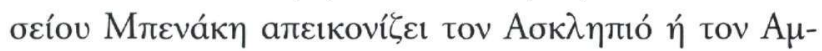

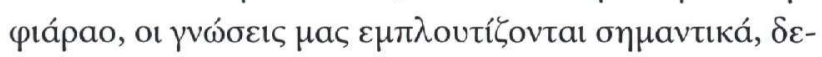

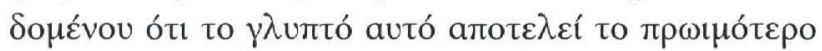

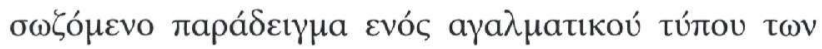

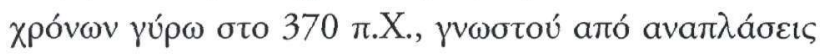

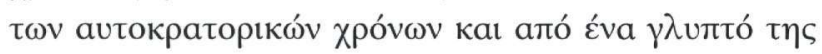

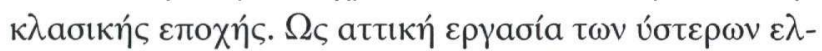

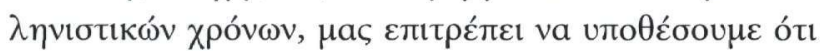

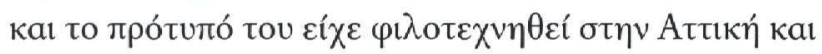

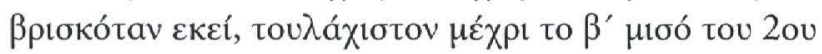

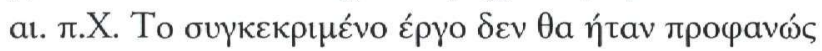

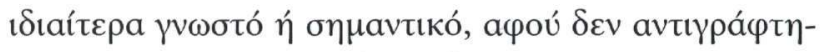

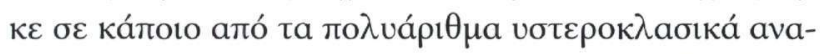

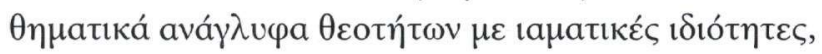

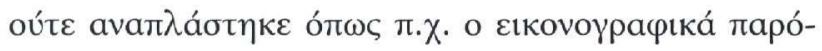

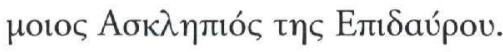

